\title{
A Review of Dynamical Studies of Multiple Stars
}

\author{
ROBERT S. HARRINGTON \\ U. S. Naval Observatory, Washington, DC 20392, USA
}

This is not going to be an exhaustive review of all of the literature on the subject of multiple star dynamics, although I will call your attention to the very recent review by Valtonen \& Mikkola (1991) entitled "The Few-Body Problem in Astrophysics". After all, from a dynamical point of view a multiple-star system is the physical realization of the n-body problem of celestial mechanics, and an enormous literature exists for that subject. Rather, this will be some personal thoughts on aspects of the few-body problem that are particularly relevant to the multiple-star astronomer.

One should not forget the case of $n=2$. Although the problem is completely solvable and contains no surprises, it is one of our reasons to exist. Especially if a binary is on a photographic program, which can yield a parallax, a total mass, and a mass-ratio, and if photometry exists, as is usually the case now for parallax stars, a real contribution is possible. Do an orbit, which is the dynamical problem, and you have two more good points for the empirical mass/luminosity relationship, which is not a bad day's work for the astrophysicist.

Go up only to $n=3$ and the situation becomes rich, complex, and completely unsolvable in the general case. In fact, going to higher $n$ does not qualitatively change the picture, and only the triple-star case will be considered for the rest of the discussion. This case can be broken into two more specific cases, those known as the hierarchical cases, and those known as the trapezium cases. These can also be called the analytical and the numerical cases, or the stable and the unstable cases.

For the hierarchical cases, we can make some analytical progress if we use what are known as Jacobi coordinates. Here, one set of coordinates is the position of the secondary with respect to the primary in the close binary, and the other set is the distant tertiary with respect to the center of mass of the binary. Fortunately, this is practically what is observed if the close binary is unresolved, allowing for the fact that we observed the photocenter, not the barycenter, of that unresolved binary.

The advantage of this formulation is that we have a small parameter, which is the ratio of the semima jor axis of the close binary orbit to that of the tertiarybarycenter orbit. The force function can then be written as the force function for the close binary, plus the force function for the tertiary-barycenter, plus an infinite power series involving powers of the small parameter and trigonometric functions of the mutual orientation angles. The power series starts with the second power of that small parameter, and hence only contains small terms.

There remains the question of whether the power series converges, but if it does, this is the theoretical justification for treating hierarchical systems as sets of nested binaries. To now form the equations of motion, you take partial derivatives of the force function with respect to the appropriate coordinate, and in so doing, one power of the small parameter disappears when dealing with the close binary, and thus the inner orbit shows an order-of-magnitude 
greater variation than the outer orbit. The motion in a triple star can thus be summarized at the following degrees of approximation:

\begin{tabular}{|c|c|c|}
\hline eroth order & - & a close binary with a fixed distant companion \\
\hline & & uter binary \\
\hline rder & & perturbed inner binary and an outer bina \\
\hline & & $\begin{array}{l}\text { full 3-body system with perturbed inner } \\
\text { and outer binaries }\end{array}$ \\
\hline
\end{tabular}

The next step is to treat the power series portion of the force function as the disturbing function in a variation-of-elements problem. The details of this kind of treatment would be more than a review in itself and therefore will not be covered here. To summarize the important results, if you use what are known as canonical elements and approach this as a problem in Hamiltonian mechanics, certain transformations are possible that lead to the result that both semimajor axes can be written as constants, plus trigonometric power series in our same small parameter. Hence, with no secular variation in the semimajor axes, these kinds of triple systems are dynamically stable, again as long as the power series converge. Since this is true in both directions in time, this means a system can not evolve from an n-body trapezium system to an n-body hierarchical system with the same $n$.

To second order, as mentioned before, the motion of the tertiary is strictly 2-body, and a solution for the motion in the binary is possible. A particularly interesting result emerges if the plane of the binary orbit is essentially perpendicular to the plane of the tertiary. To put it another way, consider the case in which the length of the total angular momentum vector of the triple is equal to the length of the angular momentum vector of the tertiary-barycenter (which is constant to this order), a situation only possible when the mutual inclination is slightly greater than a right angle. If the orientations of these two vectors come close to coincidence, as they will periodically, the length of the binary angular momentum vector can become arbitrarily small. This length contains the periastron distance as a factor, and, since the semimajor axis remains constant, the eccentricity must become close to unity.

This, of course, is no mathematical problem, but the physical consequences can best be illustrated with a particular case. The system $B D+66^{\circ} 42$, also known as ADS 440, actually is a triple, with the A component having an unseen astrometric companion. If the nodes of the two orbits coincide, the orbits are almost coplanar and there is no problem. If the nodes are opposite, the mutual inclination is 96 degrees, and the periastron distance would have been one solar radius just 11,000 years ago. To say the least, this is unlikely, and the oppositenodes case can fairly confidently be ruled out in this case.

There is now the practical question of implementing the 3-body theory for those few systems where this is necessary. Both because of the increasing timespan over which these systems have been observed and the increasing accuracy to which they can be observed, this will become important for many systems soon. No matter what approach you take, these systems will require one more orbital parameter than we are used to, and that is an epoch of osculation. Hence, this is another plea to start the practice of including this parameter in reported orbits. Again, if this has been approximated by a 2-body differential correction, this epoch is merely the weighted mean epoch. 
These effects can be included by a theory for the variation of the elements. However, the canonical ones mentioned above are hard to use, since they refer to the invariable plane, not the plane of the sky. Several authors, many of whom are at this meeting, have put forward various formulations that can be directly applied to the observed coordinate system. These all have to make some approximation, such as taking only the first term in the disturbing function, a step which is completely justifiable at today's levels of accuracy. However, if you want to be completely rigorous, a numerical approach is the only possibility. Efficient numerical integrators are easy to implement these days, and an example of our application of this approach to the Zeta Cancri system is present elsewhere in these Proceedings.

By contrast to what been discussed so far, the only way to study trapeziumtype systems is by numerical simulation. That is, you carry out numerical integrations of various specific 3-body systems and try to establish the patterns. Nothing can ever be mathematically proven this way, but a lot can still be learned. This is more like an experimental science, and these are often referred to as numerical experiments. Usually each component is referred to the center of mass of the system and is carried as part of the integration. This does not take account of the conservation of linear momentum, but it is very easy to implement, and it is easy to generalize to larger systems.

This is the version of the three-body problem of celestial mechanics that has been studied so long and so hard by very many people over very many years. Usually it is mathematical properties that are being sought, the favorite being periodic orbits. Also there are things like capture cross-section, breakup mechanism, asymptotic behavior, and the like. However, there are a few questions of specific interest to the double-star community. Particularly active in exploring these question has been the group at St. Petersburg, and of their many papers I will only mention the a good review paper by Anasova (1986).

An obvious first question to ask is what does it take to make a stable triple. What are the key factors necessary to make the series referred to earlier actually converge and produce constant semimajor axes? Stability here means just that, that there are no large variations in the axes or eccentricities, without being too specific about what is meant by large. This is often referred to as orbital stability, in contrast to some more specific stabilities employed by the mathematicians.

I discussed this at some length at the Oaxtepec meeting (see Franz \& Pismis, 1977), so I won't go into a lot of detail. It turns out that the key quantity is the ratio of the semimajor axis of the close binary to the periastron distance of the tertiary. This makes sense, since, for the high-frequency binary, you would expect sort of an averaging, whereas the tertiary exerts most of its influence when it is close to the binary. This ratio does not depend on orientation, apart from not needing to be quite as small for retrograde orbits as for direct ones, with the separation between the two cases being the perpendicular instability discussed earlier. For the equal mass case, this ratio is around $1 / 3$ and it needs to be smaller logarithmically with the mass of the tertiary. Ironically, this is just the ratio used by many people to classify observed systems, based purely on convenience and what seemed reasonable.

Perhaps related to the question of stability is the question of just what goes on when an unstable triple breaks up, an issue of particular interest to the 
St. Petersburg group. Extensive simulations have revealed the not unsurprising result that a true triple close approach is required. That is, all three stars must more or less simultaneously come very close together to permit enough interaction for one star to acquire enough energy to escape, leaving behind a more tightly bound binary.

Then there are the statistics of what is left behind after the unstable triple dissolves. The old classical result states that the probability of escape goes as the inverse third power of the mass. This would imply that single stars would tend to be less massive and therefore of later spectral types than components of binaries, which is clearly not the case. However, more recent work, again primarily at St. Petersburg shows this to not always be the case, and in certain classes of problems the escape probability is independent of mass.

The probability of a given eccentricity in the remaining binary goes linearly with the eccentricity all the way to unity, superficially counter to the observed situation. However, the observed eccentricity distribution is obviously plagued with observational selection, in that high eccentricity binaries have a much higher probability of being in a slow part of their orbit and thus not having enough motion for a decent orbit solution. Simulations have shown that this effect can completely account for the differences between observed and theoretical eccentricity distributions.

One particular reason for studying systems with more than three components is to look at the distribution of the number of components in the resulting stable systems. This requires running an enormous number of experiments to get reliable results, an effort yet to be undertaken. However, preliminary results are encouraging, in that the number of stable triples is on the order of 20 percent of the number of binaries, and stable quadruples do result from the decay of unstable quintuples, consistent with what we observe.

Abt brought up an interesting question in the scientific session of Commission 26 in Buenos Aires, and that is the maximum age that can be expected in trapezium systems. This question has already been addressed in some detail by the cometary dynamics people for our solar system. Since relative motion is determined by the sum of masses, not the product, the problem of a comet going around the Sun is the same as the problem of the dynamics of a binary star. The significant perturbation for the very wide component that has almost escaped is not the multiple nature of what is left behind, but the general tidal potential of the galaxy. More specifically, it is, by more than an order of magnitude, the tidal potential perpendicular to the galactic disk, not the central radial tidal potential. The maximum size of the Oort cloud or a stable multiple system is just over one light-year, and the associated periods would be a few tens of millions of years. Thus, any trapezium system would have to dissolve in such periods, a result in complete agreement with the observational result from a detailed analysis of the few systems discussed by Abt.

While largely an area of interest to the mathematicians of celestial mechanics, the results of the n-body problem can be applied to our understanding of multiple stars. The meeting in Oaxtepec in 1975 (Franz \& Pismis 1977) revealed this in a major way, and several groups are now active in this area. We can look forward to many useful and interesting results. 


\section{REFERENCES}

Anasova, J.P. 1986, “Dynamical Evolution of Triple Systems”, Ap\&SS, 124, 217

Frang, O.G. \& Pismis, P. 1977, eds. Observational Parameters and Dynamical Evolution of Multiple Stars, IAU Colloquium No. 33, Rev. Mex. Astron. y Astrofis., 3

Valtonen, M. \& Mikkola, S. 1991, "The Few-Body Problem in Astrophysics", ARA EA, 29, 9

\section{DISCUSSION}

ARMSTRONG: Why don't trapezium systems evolve to hierarchical systems? I can understand the reverse.

HARRINGTON: Because of the dynamical reversibility of triple systems, the result of constant semi-major axis extends infinitely back into the past as well as forward into the future. 